\title{
Uncertainty estimation for improving accuracy of non-rigid registration in cardiac images
}

\begin{abstract}
In order to utilize both computed tomography (CT) and echocardiography images of the heart for medical applications such as diagnosis and image guided intervention concurrently, nonrigid registration is an essential task. A challenging but important problem in image registration is evaluating the performance of a registration algorithm. The direct quantitative approach is to compare the deformation field solution with the ground truth transformation (at all or some landmark pixels). However, in clinical data, the ground truth is typically unknown. To deal with the absence of ground truth, some methods opted to estimate registration accuracy by using uncertainty measures as a surrogate for quantitative registration error. In this paper, we define the registration uncertainty and explore its use for diagnostic purposes. We use uncertainty estimation for improving accuracy of a hybrid registration which register a pre-operative CT to an intra-operative echocardiography images. In other words, uncertainty estimation is used to evaluate the registration algorithm performance which integrates intensity-based and feature-based methods. This registration can potentially be used to improve the diagnosis of cardiac disease by augmenting echocardiography images with high-resolution CT images and to facilitate intra-operative image fusion for minimally invasive cardio-thoracic surgical navigation. Here, we show how to determine the registration uncertainty, by using uncertainty quantification regarding to abnormal intensity and geometry distribution. The result indicates that registration uncertainty is a good predictor for the functional abnormality of subjects.
\end{abstract}

Keywords: Uncertainty; Multimodality image registration; Hybrid featurebased; Intensitybased echocardiography; Computed tomography (CT) 